\title{
Non-equilibrium Thermodynamic Extension of the Phenomenological Theories of First-order Phase Transitions
}

\author{
${ }^{1}$ Shutao Ai*, ${ }^{2}$ Yuanzhen Cai \\ ${ }^{1}$ Shool of Science, Linyi University, Linyi 276012, People's Republic of China \\ ${ }^{2}$ Library, Linyi University, Linyi 276012, People's Republic of China \\ E-mail: ${ }^{1}$ aist@1yu.edu.cn, ${ }^{2}$ caiyuanzhen@1yu.edu.cn
}

\begin{abstract}
Because the phenomenological theories of phase transitions which are based on the equilibrium thermodynamics cannot describe the first-order phase transition processes accurately, the non-equilibrium thermodynamics was applied to extending the existing phenomenological theories of first-order phase transitions. First, the internal interactions of system at a first-order phase transition were considered. The nominal stress, the nominal volume force, the internal electric field and the internal magnetic field were introduced to characterize them. Then, the most general Gibbs equation except the factor of chemical reactions was established. Based on the conservation of energy and the transformation between internal energy and kinetic energy, the rate of local entropy production was deduced. Then based on the principle of minimum entropy production and the generalized Onsager reciprocal relations, the local, evolving characteristics of a first-order phase transition (e.g. a first-order ferroelectric phase transition) were described well. It makes up the inadequateness of the older phenomenological theories.
\end{abstract}

\section{Keywords: First-order phase transition; phenomenological theory; non-equilibrium thermodynamics; internal field.}

\section{Introduction}

It is well known that the phase transitions are studied theoretically from the macroscopic and microscopic points of view, respectively. The studies from the macroscopic point of view involve the correlations of phenomena only but not the microscopic nature. So the corresponding theories are called "phenomenological" theories. Generally speaking, they include two parts: one is the phase transition thermodynamics and the other is the phase transition dynamics [1-3]. Here, we pay attention to the former one only.

The existing theories of phase transition thermodynamics, varying from the Clausius-Clapeyron equation that describes the first-order phase transitions to the Landau theory for continuous phase transitions, are limited in the range of equilibrium thermodynamics $[1,2]$. The appearances of phase transitions show that the fundamental distinctions between the first-order and the continuous phase transitions are having the latent heat or not and the coexisting of two phases or not. Here, we emphasize such a factor that the phase transitions occurring in the nature or laboratories are often achieved by altering the temperatures of systems. Then, the existence of latent heat or not and the transfer of latent heat in system will make differences of thermodynamic pictures between the first-order and the continuous phase transitions. For the former, the systems are out equilibrium, and for the latter, the systems are in equilibrium.

So, we should apply the non-equilibrium thermodynamics to processing the first-order phase transitions. In addition, the theories and methods of nonequilibrium thermodynamics have been applied to many problems successfully $[4,5]$. But it is a pity that the Landau theory for continuous phase transitions was generalized unrealistically to process the first-order phase transitions (especially the first-order ferroelectric phase transitions) instead of applying the non-equilibrium thermodynamics to the first-order phase transitions directly [6, 7]. The later study reveals that though this generalization is successful in mathematics the real physical processes are distorted [8]. Moreover, the whole process of a first-order phase transition can be regarded as a stationary-states-process mentioned often in the non-equilibrium thermodynamics as long as the phase transition is achieved by the quasi-static heating or cooling [8].

One goal of this article is to study how to apply the nonequilibrium thermodynamics to describing the first-order phase transitions on the basis of the classical phase transition thermodynamics so as to obtain a more objective physical picture of phase transitions. Meanwhile, we examine the reliability of the utilized theoretical method and look for new possible methods.

\section{Processing of Internal Fields}

The common first-order phase transitions include the solid-liquid phase transitions, the liquid-vapor phase transitions, the partial ferroelectric or ferroelastic phase transitions, the superconducting phase transitions affected by an external magnetic field, and the martensite transformations, etc. A new standard can be adopted to classify so many first-order phase transitions: the first-order phase transitions involve electromagnetic effects and the other first-order phase transitions that do not involve electromagnetic effects. Then, the solid-liquid phase transitions, the liquid-vapor phase transitions, the ferroelastic phase transitions and the martensite transformations belong to the latter, while the ferroelectric phase transitions, the superconducting phase transitions affected by an external magnetic field belong to the former. By the way, the ferromagnetic phase transitions do not 
belong to the first-order phase transitions at all but to the second-order phase transitions.

In experiments, it is discovered that the occurrence of whichever of first-order phase transitions is accompanied with the changes of the system's volume and shape, more or less. Even the condensation states of matter will change for the solid-liquid and liquid-vapor phase transitions. The changes of the system's volume and shape should be studied in the range of mechanics. The mechanics of continuous media has given us such a physical picture that the stresses and the strains are correlated by some certain constitutive relations. If there are no the strains, the stresses do not exist. But this is the picture without the occurrences of phase transitions. If a phase transition occurs, the picture changes. There is no harm in considerations as the follows. After a phase transition finishes, the volume and shape of the system change, i.e., the strain comes into being. But the system is mechanically-free, i.e., there is no the stress. On the contrary, there are some strong internal actions which force the system's volume and shape to change though there is no the strain when the phase transition begins. The actions exist in the system and should be regarded as some "internal" fields. There is no harm in characterizing the internal fields by using the concepts of stress and volume force, i.e., regarding them as the nominal stress and volume force. The sums of the nominal and the real stresses (or volume forces) give out the general mechanical effects, i.e.,

$\boldsymbol{\sigma}=\boldsymbol{\sigma}^{\text {real }}+\boldsymbol{\sigma}^{\text {nom }}$

$\boldsymbol{F}=\boldsymbol{F}^{\text {real }}+\boldsymbol{F}^{\text {nom }}$

where $\boldsymbol{\sigma}, \boldsymbol{F}$ is the stress, the volume force (per unit mass), respectively; $\boldsymbol{\sigma}^{\text {real }}, \boldsymbol{\sigma}^{\text {nom }}, \boldsymbol{F}^{\text {real }}, \boldsymbol{F}^{\text {nom }}$ is the real stress, the nominal stress, the real volume force (per unit mass) and the nominal volume force (per unit mass), respectively. $\boldsymbol{\sigma}^{\text {nom }}$ and $\boldsymbol{F}^{\text {nom }}$ are not zero when the phase transition begins but reduce to zero when the phase transition finishes.

For the first-order phase transitions which involve electromagnetic effects, besides the mechanically-internal fields there are the electromagnetically-internal fields. The most naive concept related to them originated from the "molecular field", which was put forward by Weiss to explain the ferromagnetism $[9,10]$. In fact, this is easy to be understood. The polarization or magnetization of system without the external electric or magnetic field in some certain temperature ranges can only be attributed to some certain internal actions, which can be equated to the electric or magnetic field existing in the system. As for their microscopic nature, we do not consider too much, here. So, we can imitate the processing of mechanical type and write

$\boldsymbol{E}=\boldsymbol{E}_{i}+\boldsymbol{E}_{e}$

$\boldsymbol{H}=\boldsymbol{H}_{i}+\boldsymbol{H}_{e}$

where $\boldsymbol{E}, \boldsymbol{H}$ is the total electric field and the total magnetic field, respectively; $\boldsymbol{E}_{\boldsymbol{i}}, \boldsymbol{E}_{\boldsymbol{e}}, \boldsymbol{H}_{\boldsymbol{i}}, \boldsymbol{H}_{\boldsymbol{e}}$ is the internal electric field, the external electric field, the internal magnetic field and the external magnetic field, respectively. Moreover, we assume that the internal electric field and the internal magnetic field are static fields, i.e., there are the internal electric potential $\varphi_{i}^{e}$ and the internal magnetic scalar potential $\varphi_{i}^{m}$ which satisfy
$\boldsymbol{E}_{i}=-\nabla \varphi_{i}^{e}$

$\boldsymbol{H}_{i}=-\nabla \varphi_{i}^{m}$

\section{Non-equilibrium Thermodynamic Extension}

There are mainly two thermodynamic approaches to deal with the irreversibility, i.e., the entropy generation approach and the entropy production approach. The former one is a global theory method, which allows us to obtain the information directly about the mean values of physical quantities [11]. It is a method for modeling the irreversible processes and devices, and can optimize the finite size and finite time constraints, and it has been developed in thermal science and engineering in order to describe the heat transfer processes and energy devices. It is based on the consideration that the destroyed power is proportional to the total rate of entropy generation. In order to optimize the efficiency of engineering systems, it is necessary to design them in such a way that the entropy generation rate is extremum. A great number of applications of this method such as $[12,13]$ have been made successfully. The latter one is a local theory method, which yields the information about the distribution of physical quantities. It is a classical one and well known to people. Here, we care for the local, evolving characteristics of first-order phase transitions, so we utilize the latter one to investigate them.

Because of the mathematical complexity of the nonequilibrium thermodynamic theories and methods and that the system is in the vicinity of "global equilibrium", we choose the primary non-equilibrium thermodynamics, i.e., the linear thermodynamics to extend the phenomenological theories of first-order phase transitions and expect to obtain a result that a better extension can be made by others.

\subsection{Establishment of Gibbs Equation}

One of the fundamental hypothesizes of the linear thermodynamics is the local equilibrium hypothesis that some local thermodynamic quantities satisfy the Gibbs equation i.e., the thermodynamic, mechanical, electromagnetic forces are small. We consider both the mechanical effects and the electromagnetic effects in order to establish the most general Gibbs equation except the factor of chemical reactions.

First, let's consider the work done by the mechanical forces. The mechanical power can be written as

$\frac{\mathrm{d} W}{\mathrm{~d} t}=\oiint_{\Sigma(t)} \boldsymbol{\sigma}_{n} \cdot \boldsymbol{v} \mathrm{d} \Sigma+\iiint_{V(t)} \rho \boldsymbol{F} \cdot \boldsymbol{v} \mathrm{d} V$

where $W$ is the work done by the mechanical forces, $t$ is the time, $\sigma_{\boldsymbol{n}}$ is the surface force, $\boldsymbol{v}$ is the velocity, $\mathrm{d} \Sigma$ is the surface element, $\rho$ is the mass density, $\boldsymbol{F}$ is the volume force exerted on unit mass, $\mathrm{d} V$ is the volume element. Let's pay attention to the first term on the right side of Eq. (7) and we deduce

$$
\begin{aligned}
& \boldsymbol{\sigma}_{n} \cdot \boldsymbol{v} \mathrm{d} \Sigma=\boldsymbol{v} \cdot \boldsymbol{\sigma}_{n} \mathrm{~d} \Sigma=\boldsymbol{v} \cdot(\boldsymbol{\sigma} \cdot \boldsymbol{n}) \mathrm{d} \Sigma=(\boldsymbol{v} \cdot \boldsymbol{\sigma}) \cdot \boldsymbol{n} \mathrm{d} \Sigma=\boldsymbol{v} \\
& \boldsymbol{\sigma} \cdot \mathrm{d} \boldsymbol{\Sigma}
\end{aligned}
$$

where the stress tensor $\boldsymbol{\sigma}$ relates the pressure tensor $\boldsymbol{P}$ by $\boldsymbol{\sigma}=-\boldsymbol{P}$ when the matter is fluid, $\mathrm{d} \boldsymbol{\Sigma}$ is the directed surface element, $\boldsymbol{n}$ is the unit normal vector directed outwards. The mechanical power done for unit mass can be obtained by using the Gaussian formula 
$p=\frac{1}{\rho} \nabla \cdot(\boldsymbol{v} \cdot \boldsymbol{\sigma})+\boldsymbol{F} \cdot \boldsymbol{v}$

Second, let us consider the work done by the electromagnetic fields. Generally speaking, the electromagnetic fields make the media polarized or magnetized or conduct the electric current. So, there are three terms related to the corresponding work (per unit mass)

$\frac{1}{\rho} \boldsymbol{E} \cdot \mathrm{d} \boldsymbol{D}+\frac{1}{\rho} \boldsymbol{H} \cdot \mathrm{d} \boldsymbol{B}+\frac{1}{\rho} \boldsymbol{E} \cdot \boldsymbol{J} \mathrm{d} t$

where $\boldsymbol{D}, \boldsymbol{B}, \boldsymbol{J}$ is the electric displacement, the magnetic induction and the conduction current density, respectively. Moreover, we pay attention to that the magnetic field does not do work to the electric current so there is no the corresponding term related to the work.

So, we obtain the most general Gibbs equation except the factor of chemical reactions

$T \mathrm{~d} s+\sum_{i} \mu_{i} \mathrm{~d} n_{i}+\frac{1}{\rho} \boldsymbol{E} \cdot \mathrm{d} \boldsymbol{D}+\frac{1}{\rho} \boldsymbol{H} \cdot \mathrm{d} \boldsymbol{B}+\frac{1}{\rho} \boldsymbol{E} \cdot \boldsymbol{J} \mathrm{d} t+\boldsymbol{F} \cdot$

$\boldsymbol{v} \mathrm{d} t+\frac{1}{\rho} \nabla \cdot(\boldsymbol{v} \cdot \boldsymbol{\sigma}) \mathrm{d} t=\mathrm{d} u+\mathrm{d} k$

where $T$ is the temperature, $s$ is the entropy density (per unit mass), $u$ is the internal energy density (per unit mass), $k=v^{2} / 2$ is the kinetic energy density (per unit mass), $n_{i}$ is the molar number density (per unit mass), $\mu_{i}$ is the chemical potential.

\subsection{Local Entropy Production}

The local entropy balance equation can be deduced from the Gibbs equation, i.e., Eq.(11) by considering the conservation of energy and the transformation between kinetic energy and internal energy (see Appendix)

$\rho \frac{\mathrm{d} s}{\mathrm{~d} t}=-\nabla \cdot \boldsymbol{J}_{s}+\sigma_{s}$

where the entropy flux

$\boldsymbol{J}_{S}=\frac{\boldsymbol{J}_{q}^{\text {diff }}+\varphi^{e} \boldsymbol{J}_{P}+\varphi^{m} \boldsymbol{J}_{M}+\varphi^{e} \boldsymbol{J}-\boldsymbol{v} \cdot \boldsymbol{\sigma}}{T}$

and the local entropy production is

$\sigma_{s}=\boldsymbol{J}_{q}^{\text {diff }} \cdot \nabla\left(\frac{1}{T}\right)-\sum_{i} \boldsymbol{J}_{n i}^{\text {diff }} \cdot \frac{\nabla \mu_{i}}{T}+\boldsymbol{J}_{P} \cdot \nabla\left(\frac{\varphi^{e}}{T}\right)+\boldsymbol{J}_{M}$.

$\nabla\left(\frac{\varphi^{m}}{T}\right)+\boldsymbol{J} \cdot \nabla\left(\frac{\varphi^{e}}{T}\right)-\boldsymbol{\sigma}:\left[\boldsymbol{v} \nabla\left(\frac{1}{T}\right)\right]-\rho \boldsymbol{F} \cdot \frac{\boldsymbol{v}}{T}$

In the two equations above, $\boldsymbol{J}_{q}^{\text {diff }}$ is the diffusion part of heat flux, i.e., the heat conduction, $\varphi^{e}$ is the electric potential, $\varphi^{m}$ is the magnetic scalar potential, $\boldsymbol{J}_{P}$ is the polarization current (in form), $\boldsymbol{J}_{M}$ is the magnetic charges current (in form), $\boldsymbol{J}_{n i}^{\text {diff }}$ is the diffusion part of particle current. In Eq. (14), the independent fluxes are $\boldsymbol{J}_{q}^{\text {diff }}, \boldsymbol{J}_{n i}^{\text {diff }}$, $\boldsymbol{J}_{P}+\boldsymbol{J}, \boldsymbol{J}_{M}, \boldsymbol{\sigma}$ and $\rho \boldsymbol{F}$; and the corresponding independent forces are $\nabla\left(\frac{1}{T}\right),-\frac{\nabla \mu_{i}}{T}, \nabla\left(\frac{\varphi^{e}}{T}\right), \nabla\left(\frac{\varphi^{m}}{T}\right),-\boldsymbol{v} \nabla\left(\frac{1}{T}\right)$ and $-\frac{v}{T}$.

In fact, from the classical point of view of physics, we know that the polarization of media and the electric conductance of those are repellent each other. So, $\boldsymbol{J}_{P}$ and $\boldsymbol{J}$ can't come into being simultaneously. Generally speaking, no electric field is exerted on the conductor which is in an external magnetic field. The ordinary state of conductor changes to be the super-conduction state with the decrease of temperature. So, neither $\boldsymbol{J}_{P}$ nor $\boldsymbol{J}$ can come into being. The second term on the right hand of Eq. (14) should be considered when one processes the phase transitions controlled by the diffusion of particles. Nevertheless, it need not to be considered for the phase transitions which are not controlled by the diffusion of particles (e.g. the ferroelectric phase transitions).

\subsection{Description of First-order Phase Transitions (e.g. Ferroelectric Phase Transitions)}

Because the ferroelectric phase transitions involve both the mechanical effects and the electromagnetic effects, we study how to process the first-order phase transitions, e.g., the first-order ferroelectric phase transitions by using the non-equilibrium thermodynamics. To get the common knowledge of ferroelectrics, one may refer to [6]. Assume there are no the external electromagnetic fields exerted on the system, i.e., the phase transition is affected by the internal electric field only, the local entropy production, i.e., Eq. (14) can be reduced to

$$
\sigma_{s}=\boldsymbol{J}_{q}^{\text {diff }} \cdot \nabla\left(\frac{1}{T}\right)+\boldsymbol{J}_{P} \cdot \nabla\left(\frac{\varphi_{i}^{e}}{T}\right)-\boldsymbol{\sigma}:\left[\boldsymbol{v} \nabla\left(\frac{1}{T}\right)\right]-\rho \boldsymbol{F} \cdot \frac{\boldsymbol{v}}{T}
$$

where the thermodynamic fluxes $\boldsymbol{J}_{q}^{\text {diff }}, \boldsymbol{J}_{P}, \boldsymbol{J}_{\sigma}(=\boldsymbol{\sigma}), \boldsymbol{J}_{F}(=$ $\rho \boldsymbol{F})$ and the corresponding thermodynamic forces $\boldsymbol{X}_{q}, \boldsymbol{X}_{P}$, $\boldsymbol{X}_{\sigma}, \boldsymbol{X}_{F}$ are

$\boldsymbol{X}_{q}=\nabla\left(\frac{1}{T}\right)$

$\boldsymbol{X}_{P}=\nabla\left(\frac{\varphi_{i}^{e}}{T}\right)$

$\boldsymbol{X}_{\sigma}=-\boldsymbol{v} \nabla\left(\frac{1}{T}\right)$

$\boldsymbol{X}_{F}=-\frac{v}{T}$

$\boldsymbol{J}_{i}(i=q, P, \sigma, F)$ can be expanded linearly with $\boldsymbol{X}_{j}(j=$ $q, P, \sigma, F)$

$\boldsymbol{J}_{q}^{\text {diff }}=\boldsymbol{L}_{q q} \cdot \boldsymbol{X}_{q}+\boldsymbol{L}_{q P} \cdot \boldsymbol{X}_{P}+\boldsymbol{L}_{q \sigma}: \boldsymbol{X}_{\sigma}+\boldsymbol{L}_{q F} \cdot \boldsymbol{X}_{F}$

$\boldsymbol{J}_{P}=\boldsymbol{L}_{P q} \cdot \boldsymbol{X}_{q}+\boldsymbol{L}_{P P} \cdot \boldsymbol{X}_{P}+\boldsymbol{L}_{P \sigma}: \boldsymbol{X}_{\sigma}+\boldsymbol{L}_{P F} \cdot \boldsymbol{X}_{F}$

$\boldsymbol{J}_{\sigma}=\boldsymbol{L}_{\sigma q} \cdot \boldsymbol{X}_{q}+\boldsymbol{L}_{\sigma P} \cdot \boldsymbol{X}_{P}+\boldsymbol{L}_{\sigma \sigma}: \boldsymbol{X}_{\sigma}+\boldsymbol{L}_{\sigma F} \cdot \boldsymbol{X}_{F}$

$\boldsymbol{J}_{F}=\boldsymbol{L}_{F q} \cdot \boldsymbol{X}_{q}+\boldsymbol{L}_{F P} \cdot \boldsymbol{X}_{P}+\boldsymbol{L}_{F \sigma}: \boldsymbol{X}_{\sigma}+\boldsymbol{L}_{F F} \cdot \boldsymbol{X}_{F}$

where $\boldsymbol{L}_{q q}, \boldsymbol{L}_{P P}, \boldsymbol{L}_{F F}, \boldsymbol{L}_{q P}, \boldsymbol{L}_{P q}, \boldsymbol{L}_{q F}, \boldsymbol{L}_{F q}, \boldsymbol{L}_{P F}, \boldsymbol{L}_{F P}$ are nine second-order tensors, $\boldsymbol{L}_{q \sigma}, \boldsymbol{L}_{\sigma q}, \boldsymbol{L}_{P \sigma}, \boldsymbol{L}_{\sigma P}, \boldsymbol{L}_{F \sigma}, \boldsymbol{L}_{\sigma F}$ are six third-order tensors, and $\boldsymbol{L}_{\sigma \sigma}$ is a fourth-order tensor. Then the local entropy production can be written as

$\sigma_{S}=\boldsymbol{L}_{q q}: \boldsymbol{X}_{q} \boldsymbol{X}_{q}+\boldsymbol{L}_{q P} \cdot \boldsymbol{X}_{P} \boldsymbol{X}_{q}+\boldsymbol{L}_{q \sigma} \cdots \boldsymbol{X}_{\sigma}^{T} \boldsymbol{X}_{q}+\boldsymbol{L}_{q F} \cdot \cdot$

$\boldsymbol{X}_{F} \boldsymbol{X}_{q}+\boldsymbol{L}_{P q} \cdot \boldsymbol{X}_{q} \boldsymbol{X}_{P}+\boldsymbol{L}_{P P}: \boldsymbol{X}_{P} \boldsymbol{X}_{P}+\boldsymbol{L}_{P \sigma} \cdots \boldsymbol{X}_{\sigma}^{T} \boldsymbol{X}_{P}+\boldsymbol{L}_{P F} \cdot \cdot$

$\boldsymbol{X}_{F} \boldsymbol{X}_{P}+\boldsymbol{L}_{\sigma q} \cdots \boldsymbol{X}_{q} \boldsymbol{X}_{\sigma}^{T}+\boldsymbol{L}_{\sigma P} \cdots \boldsymbol{X}_{P} \boldsymbol{X}_{\sigma}^{T}+\boldsymbol{L}_{\sigma \sigma} \cdots \boldsymbol{X}_{\sigma}^{T} \boldsymbol{X}_{\sigma}^{T}+$

$\boldsymbol{L}_{\sigma F} \cdots \boldsymbol{X}_{F} \boldsymbol{X}_{\sigma}^{T}+\boldsymbol{L}_{F q} \cdot \boldsymbol{X}_{q} \boldsymbol{X}_{F}+\boldsymbol{L}_{F P} \cdot \boldsymbol{X}_{P} \boldsymbol{X}_{F}+$

$\boldsymbol{L}_{F \sigma} \cdots \boldsymbol{X}_{\sigma}^{T} \boldsymbol{X}_{F}+\boldsymbol{L}_{F F}: \boldsymbol{X}_{F} \boldsymbol{X}_{F}$ 
If the system is in a stationary state, the principle of minimum entropy production is satisfied. Yet, the phase transition process can be regarded as a stationary-statesprocess (i.e. the system can pass a series of stationary states continuously by changing the external conditions). So,

$\left(\frac{\partial \sigma_{s}}{\partial \boldsymbol{X}_{q}}\right)_{\boldsymbol{X}_{P} \boldsymbol{X}_{\sigma}, \boldsymbol{X}_{F}}=2 \boldsymbol{L}_{q q} \cdot \boldsymbol{X}_{q}+\boldsymbol{L}_{q P} \cdot \boldsymbol{X}_{P}+\boldsymbol{L}_{q \sigma}: \boldsymbol{X}_{\sigma}+\boldsymbol{L}_{q F} \cdot$

$\boldsymbol{X}_{F}+\boldsymbol{L}_{P q}^{T} \cdot \boldsymbol{X}_{P}+\boldsymbol{L}_{\sigma q}^{T}: \boldsymbol{X}_{\sigma}+\boldsymbol{L}_{F q}^{T} \cdot \boldsymbol{X}_{F}=2\left(\boldsymbol{L}_{q q} \cdot \boldsymbol{X}_{q}+\boldsymbol{L}_{q P} \cdot\right.$

$\left.\boldsymbol{X}_{P}+\boldsymbol{L}_{q \sigma}: \boldsymbol{X}_{\sigma}+\boldsymbol{L}_{q F} \cdot \boldsymbol{X}_{F}\right)=2 \boldsymbol{J}_{q}^{\text {diff }}=0$

$\left(\frac{\partial \sigma_{S}}{\partial \boldsymbol{X}_{P}}\right)_{\boldsymbol{X}_{q}, \boldsymbol{X}_{\sigma}, \boldsymbol{X}_{F}}=\boldsymbol{L}_{q P}^{T} \cdot \boldsymbol{X}_{q}+\boldsymbol{L}_{P q} \cdot \boldsymbol{X}_{q}+2 \boldsymbol{L}_{P P} \cdot \boldsymbol{X}_{P}+$

$\boldsymbol{L}_{P \sigma}: \boldsymbol{X}_{\sigma}+\boldsymbol{L}_{P F} \cdot \boldsymbol{X}_{F}+\boldsymbol{L}_{\sigma P}^{T}: \boldsymbol{X}_{\sigma}+\boldsymbol{L}_{F P}^{T} \cdot \boldsymbol{X}_{F}=2\left(\boldsymbol{L}_{P q} \cdot \boldsymbol{X}_{q}+\right.$

$\left.\boldsymbol{L}_{P P} \cdot \boldsymbol{X}_{P}+\boldsymbol{L}_{P \sigma}: \boldsymbol{X}_{\sigma}+\boldsymbol{L}_{P F} \cdot \boldsymbol{X}_{F}\right)=2 \boldsymbol{J}_{P}=0$

$\left(\frac{\partial \sigma_{s}}{\partial \boldsymbol{X}_{\sigma}}\right)_{\boldsymbol{X}_{q}, \boldsymbol{X}_{P}, \boldsymbol{X}_{F}}=\boldsymbol{L}_{q \sigma}^{T} \cdot \boldsymbol{X}_{q}+\boldsymbol{L}_{P \sigma}^{T} \cdot \boldsymbol{X}_{P}+\boldsymbol{L}_{\sigma q} \cdot \boldsymbol{X}_{q}+\boldsymbol{L}_{\sigma P} \cdot$

$\boldsymbol{X}_{P}+2 \boldsymbol{L}_{\sigma \sigma}: \boldsymbol{X}_{\sigma}+\boldsymbol{L}_{\sigma F} \cdot \boldsymbol{X}_{F}+\boldsymbol{L}_{F \sigma}^{T} \cdot \boldsymbol{X}_{F}=2\left(\boldsymbol{L}_{\sigma q} \cdot \boldsymbol{X}_{q}+\right.$

$\left.\boldsymbol{L}_{\sigma P} \cdot \boldsymbol{X}_{P}+\boldsymbol{L}_{\sigma \sigma}: \boldsymbol{X}_{\sigma}+\boldsymbol{L}_{\sigma F} \cdot \boldsymbol{X}_{F}\right)=2 \boldsymbol{J}_{\sigma}=0$

$\left(\frac{\partial \sigma_{S}}{\partial \boldsymbol{X}_{F}}\right)_{\boldsymbol{X}_{q}, \boldsymbol{X}_{P}, \boldsymbol{X}_{\sigma}}=\boldsymbol{L}_{q F}^{T} \cdot \boldsymbol{X}_{q}+\boldsymbol{L}_{P F}^{T} \cdot \boldsymbol{X}_{P}+\boldsymbol{L}_{\sigma F}^{T}: \boldsymbol{X}_{\sigma}+\boldsymbol{L}_{F q} \cdot$

$\boldsymbol{X}_{q}+\boldsymbol{L}_{F P} \cdot \boldsymbol{X}_{P}+\boldsymbol{L}_{F \sigma}: \boldsymbol{X}_{\sigma}+2 \boldsymbol{L}_{F F} \cdot \boldsymbol{X}_{F}=2\left(\boldsymbol{L}_{F q} \cdot \boldsymbol{X}_{q}+\boldsymbol{L}_{F P} \cdot\right.$

$\left.\boldsymbol{X}_{P}+\boldsymbol{L}_{F \sigma}: \boldsymbol{X}_{\sigma}+\boldsymbol{L}_{F F} \cdot \boldsymbol{X}_{F}\right)=2 \boldsymbol{J}_{F}=0$

The generalized Onsager reciprocal relations [14]

$\boldsymbol{L}_{i j}=\boldsymbol{L}_{j i}^{T}$

(the superscript " $\mathrm{T}$ " means transposition) are used in the four equations above. These extremum conditions reveal that: (i) If keep a part of thermodynamic forces to be constants, we know the thermodynamic fluxes corresponded to the rest thermodynamic forces are zero; (ii) If there are no any restrictions on all the thermodynamic forces, all the thermodynamic fluxes are zero.

We may describe a ferroelectric phase transition by using the two paradigms above. For a first-order ferroelectric phase transition, the forces $\boldsymbol{X}_{P}, \boldsymbol{X}_{\sigma}, \boldsymbol{X}_{F}$ of the region where the phase transition is occurring (i.e., a thin region adjacent to the phase boundary) can be regarded as three large constants roughly in the characteristic times of phase transition because the electric displacement, the volume and the shape change suddenly. So the flux $\boldsymbol{J}_{q}^{\text {diff }}$ of the region should be zero (but $\boldsymbol{J}_{P} \neq 0, \boldsymbol{\sigma} \neq 0, \rho \boldsymbol{F} \neq 0$ ). This states clearly that the pure heat conduction and the heat conduction induced by the thermo-electric coupling and the thermo-mechanical coupling cancel out each other so as to release or absorb the latent heat (Just see Eq. (25)). The phase transition occurs at the surface layer firstly, which is mechanically-free. So, when the phase transition occurs in this region, the flux $\boldsymbol{\sigma}$ maybe the nominal stress $\boldsymbol{\sigma}^{\text {nom }}$ only, which does work to realize the transformation from the internal energy to the kinetic energy. When the phase transition occurs in the inner part, the flux $\sigma$ should be the sum of $\boldsymbol{\sigma}^{\text {real }}$ and $\boldsymbol{\sigma}^{\text {nom }}$ because the sudden changes of the inner part's volume and shape have to overcome the bound of the outer part then $\boldsymbol{\sigma}^{\text {real }}$ arises. The region where the phase transition is occurring, is accompanied with the real stress $\boldsymbol{\sigma}^{\text {real }}$ usually, which does work to realize the transformation from the kinetic energy to the internal energy. This has been predicted and described with a propagating stress wave in [15].

It is certain that the latent heat passes through the region where the phase transition has occurred at the outside of the region and exchange itself with the thermal bath. For $\pm l \rho \boldsymbol{v}_{a}=\boldsymbol{J}_{q}^{\text {diff }}=-\boldsymbol{\kappa} \cdot \nabla T$ (where $l$ is the latent heat per unit mass, $\boldsymbol{v}_{a}$ is the average velocity of phase boundary, $\boldsymbol{\kappa}$ is the coefficient of heat conduction), a constant temperature gradient $\nabla T$ is kept in the region where the phase transition has occurred, i.e., the force $\boldsymbol{X}_{q}$ at every site is constant (which does not change with the time but may vary with the position). So, the fluxes $\boldsymbol{J}_{P}=\boldsymbol{\sigma}=$ $\rho \boldsymbol{F}=0$ (but the flux $\boldsymbol{J}_{q}^{\text {diff }} \neq 0$ ). This states clearly that the electric displacement $\boldsymbol{D}$ will not change but keep the value at the Curie temperature or zero until the phase transition finishes and $\boldsymbol{\sigma}^{\text {real }}=-\boldsymbol{\sigma}^{\text {nom }}$ in this region. Because the electric displacement $\boldsymbol{D}$ and the strain are all determined by the crystal structure of system, $\boldsymbol{J}_{P}=0$ reveals that $\boldsymbol{D}$ of this region does not change so does not the crystal structure then does not the strain. According to [15], we know the region where the phase transition has occurred is unstressed, i.e., $\boldsymbol{\sigma}^{\text {real }}=0$, then $\boldsymbol{\sigma}^{\text {nom }}=0$. This reveals that the eigen (or free) strain of system induced by the thermo-electromechanical coupling of phase transition is complete and the change of it terminates before the phase transition finishes. The two deductions coincide with each other. $\boldsymbol{\sigma}^{\text {real }}$ may relaxes via the free surface.

The region where the phase transition will occur should be in equilibrium because there are no restrictions on the forces $\boldsymbol{X}_{q}, \boldsymbol{X}_{P}, \boldsymbol{X}_{\sigma}, \boldsymbol{X}_{F}$. Whereas, according to [15], the region is stressed, i.e., $\boldsymbol{\sigma}^{\text {real }} \neq 0$. For the heating process of phase transition, this may lead to a change of spontaneous polarization of this region because of the electromechanical coupling (piezoelectric effect).

\section{Conclusions}

Because the existing phenomenological theories of firstorder phase transitions which are based on the equilibrium thermodynamics and cannot describe the non-equilibrium thermodynamic processes of phase transitions accurately, we extended the phenomenological theories by using the non-equilibrium thermodynamics. In particular, we paid attention to the "internal" fields of first-order phase transitions, which is characterized by introducing the nominal stress, the nominal volume force, the internal electric field and the internal magnetic field. We established the most general Gibbs equation except the factor of chemical reactions, and from which we deduced the most general local entropy production. The various first-order phase transitions can be processed on the basis of reducing the most general local entropy production according to the various cases. In particular, we showed the description of the local, evolving characteristics of phase transitions by using the non-equilibrium thermodynamic theory and method, which make up the inadequateness of the old phenomenological theories effectively. (On the contrary, the old theories can only describe the static, global characteristics).

The non-equilibrium thermodynamics used here is the most basic one, i.e., the linear thermodynamics. Because the forces and fluxes are large at phase transitions, the description above maybe not accurate enough. In order to get the more accurate approaches, there is no harm in using the two new theories of non-equilibrium thermodynamics: 
the thermodynamics with internal variables [16] and the extended (irreversible) thermodynamics [17]. They all expand the fundamental variables spaces to describe the irreversible processes more accurately. Whereas, the relevant theoretical processing must be more complicated without doubt. This situation needs very much effort.

\section{Acknowledgements}

We would like to thank Zeigfried Dima-Ala and Pamela Dima-Ala for their great help on English.

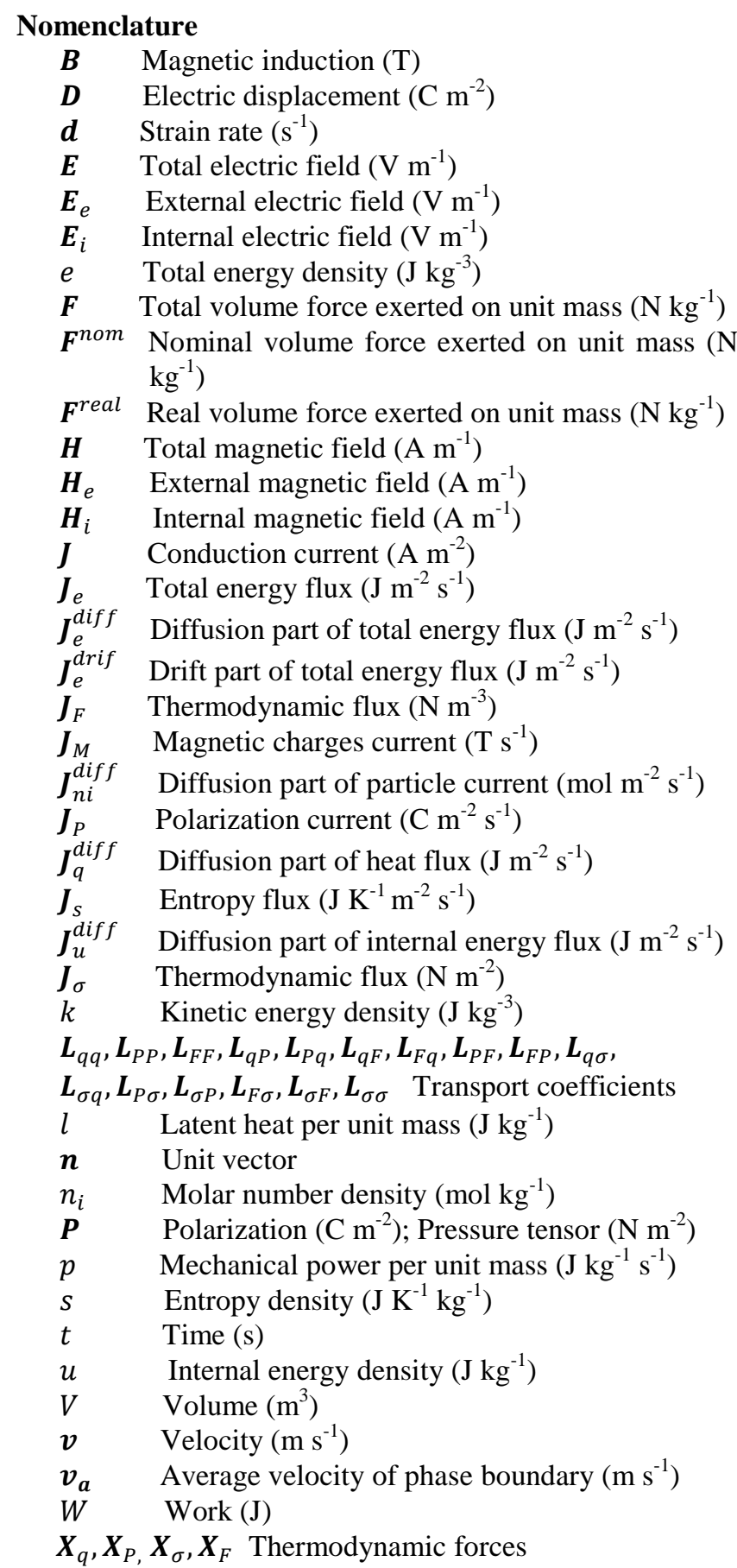

\section{Greek Symbols}

$\boldsymbol{\kappa}$ Coefficient of heat conduction $\left(\mathrm{J} \mathrm{K}^{-1} \mathrm{~m}^{-1} \mathrm{~s}^{-1}\right)$

$\mu_{i} \quad$ Chemical potential $\left(\mathrm{J} \mathrm{mol}^{-1}\right)$

$\rho \quad$ Mass density $\left(\mathrm{kg} \mathrm{m}^{-3}\right)$

$\Sigma \quad$ Area $\left(\mathrm{m}^{2}\right)$

$\boldsymbol{\sigma} \quad$ Stress $\left(\mathrm{N} \mathrm{m}^{-2}\right)$

$\sigma_{n} \quad$ Surface force $\left(\mathrm{N} \mathrm{m}^{-2}\right)$

$\boldsymbol{\sigma}^{\text {nom }}$ Nominal stress $\left(\mathrm{N} \mathrm{m}^{-2}\right)$
$\boldsymbol{\sigma}^{\text {real }}$ Real stress $\left(\mathrm{N} \mathrm{m}^{-2}\right)$
$\sigma_{q} \quad$ Heat production $\left(\mathrm{J} \mathrm{m}^{-3} \mathrm{~s}^{-1}\right)$
$\sigma_{s} \quad$ Entropy production $\left(\mathrm{J} \mathrm{K}^{-1} \mathrm{~m}^{-3} \mathrm{~s}^{-1}\right)$
$\sigma_{u} \quad$ Internal energy production $\left(\mathrm{J} \mathrm{m}^{-3} \mathrm{~s}^{-1}\right)$
$\varphi^{e} \quad$ Total electric potential (V)
$\varphi^{m} \quad$ Total magnetic scalar potential $\left(\mathrm{J} \mathrm{m}^{-2} \mathrm{~T}^{-1}\right)$
$\varphi_{i}^{e} \quad$ Internal electric potential (V)
$\varphi_{i}^{m} \quad$ Internal magnetic scalar potential $\left(\mathrm{J} \mathrm{m}^{-2} \mathrm{~T}^{-1}\right)$

\section{Superscripts}

diff Diffusion

drif Drift

$e \quad$ Electric

$m$ Magnetic

nom Nominal

real Real

T Transposition

\section{Subscripts}

$\begin{array}{ll}a & \text { Average } \\ e & \text { External; Total energy } \\ F & \text { Volume force } \\ i & \text { Internal; The i-th } \\ M & \text { Magnetic } \\ n & \text { The direction of } \boldsymbol{n} \\ n_{i} & \text { The i-th kind of particles } \\ P & \text { Polarization } \\ q & \text { Heat } \\ s & \text { Entropy } \\ u & \text { Internal energy } \\ \sigma & \text { Stress }\end{array}$

Appendix: Derivation of Local Entropy Production

In this appendix, we deduce the formula of local entropy production from Eq. (11). Make the material derivative of Eq. (11) with $t$, then obtain

$\rho \frac{\mathrm{d} s}{\mathrm{~d} t}+\frac{\rho}{T} \sum_{i} \mu_{i} \frac{\mathrm{d} n_{i}}{\mathrm{~d} t}+\frac{1}{T} \boldsymbol{E} \cdot \frac{\mathrm{d} \boldsymbol{D}}{\mathrm{d} t}+\frac{1}{T} \boldsymbol{H} \cdot \frac{\mathrm{d} \boldsymbol{B}}{\mathrm{d} t}+\frac{1}{T} \boldsymbol{E} \cdot \boldsymbol{J}+\frac{\rho}{T} \boldsymbol{F} \cdot \boldsymbol{v}+$

$\frac{1}{T} \nabla \cdot(\boldsymbol{v} \cdot \boldsymbol{\sigma})=\frac{\rho}{T} \frac{\mathrm{d} e}{\mathrm{~d} t}$

where $e=u+k$ is the total energy density (per unit mass). The equation of energy conservation in Eulerian form is

$\frac{\partial}{\partial t}(\rho e)=-\nabla \cdot J_{e}$

where $\boldsymbol{J}_{e}$ is the energy flux, which consists of two parts: the diffusion part $\boldsymbol{J}_{e}^{\text {diff }}$ and the drift part $\boldsymbol{J}_{e}^{\text {drif }}$, i.e.,

$\boldsymbol{J}_{e}=\boldsymbol{J}_{e}^{\text {diff }}+\boldsymbol{J}_{e}^{\text {drif }}$

The term on the left hand of Eq. (A2) can be rewritten as

$$
\begin{aligned}
& \frac{\partial}{\partial t}(\rho e)=\frac{\mathrm{d}}{\mathrm{d} t}(\rho e)-\boldsymbol{v} \cdot \nabla(\rho e)=e \frac{\mathrm{d} \rho}{\mathrm{d} t}+\rho \frac{\mathrm{d} e}{\mathrm{~d} t}-\boldsymbol{v} \cdot \nabla(\rho e) \\
& =-\rho e \nabla \cdot \boldsymbol{v}+\rho \frac{\mathrm{d} e}{\mathrm{~d} t}-\boldsymbol{v} \cdot \nabla(\rho e)=\rho \frac{\mathrm{d} e}{\mathrm{~d} t}-\nabla \cdot(\rho e \boldsymbol{v})
\end{aligned}
$$

where the equation of mass conservation in Lagrangian form 
$\frac{\mathrm{d} \rho}{\mathrm{d} t}+\rho \nabla \cdot \boldsymbol{v}=0$

is used. Paying attention to that $\rho e v$ in Eq. (A4) is just the drift part of energy flux $\boldsymbol{J}_{e}^{\text {drif }}$, we can obtain

$\rho \frac{\mathrm{d} e}{\mathrm{~d} t}=-\nabla \cdot \boldsymbol{J}_{e}^{\text {diff }}$

$\boldsymbol{J}_{e}^{\text {diff }}$ should consists of two parts: the diffusion of kinetic energy $-\boldsymbol{v} \cdot \boldsymbol{\sigma}$ [17] and the diffusion of the internal energy $\boldsymbol{J}_{u}^{\text {diff }}$, i.e.,

$\boldsymbol{J}_{e}^{\text {diff }}=-\boldsymbol{v} \cdot \boldsymbol{\sigma}+\boldsymbol{J}_{u}^{\text {diff }}$

$J_{u}^{\text {diff }}$ can be worked out from the following deduction. $\mathrm{d} u$ consists of six parts

$\mathrm{d} u=\mathrm{d} q+\sum_{i} \mu_{i} \mathrm{~d} n_{i}+\frac{1}{\rho} \boldsymbol{E} \cdot \mathrm{d} \boldsymbol{D}+\frac{1}{\rho} \boldsymbol{H} \cdot \mathrm{d} \boldsymbol{B}$

$+\frac{1}{\rho} \boldsymbol{E} \cdot \boldsymbol{J} \mathrm{d} t+\frac{1}{\rho} \boldsymbol{\sigma}: \boldsymbol{d} \mathrm{d} t$

where $q$ is the thermal quantity (per unit mass), $\boldsymbol{d}=$ $(\nabla v+v \nabla) / 2$ is the strain rate. Differentiating Eq. (A8) with $t$ and paying attention to that $\boldsymbol{E}=-\nabla \varphi^{e}$ (where $\varphi^{e}$ is the electric potential), $\boldsymbol{H}=-\nabla \varphi^{m}$ (where $\varphi^{m}$ is the magnetic scalar potential), the polarization current (in form) $\boldsymbol{J}_{P}=\mathrm{d} \boldsymbol{D} / \mathrm{d} t$, the magnetic charges current (in form) $\boldsymbol{J}_{M}=\mathrm{d} \boldsymbol{B} / \mathrm{d} t$, $\rho\left(\mathrm{d} n_{i} / \mathrm{d} t\right)=-\nabla \cdot J_{n i}^{\text {diff }} \quad\left(\boldsymbol{J}_{n i}^{\text {diff }}\right.$ is the diffusion part of particles current), we obtain

$\rho \frac{\mathrm{d} u}{\mathrm{~d} t}=\rho \frac{\mathrm{d} q}{\mathrm{~d} t}-\sum_{i} \mu_{i} \nabla \cdot \boldsymbol{J}_{n i}^{\text {diff }}-\nabla \varphi^{e} \cdot \boldsymbol{J}_{P}-\nabla \varphi^{m} \cdot \boldsymbol{J}_{M}$

$-\nabla \varphi^{e} \cdot \boldsymbol{J}+\frac{1}{2} \boldsymbol{\sigma}:(\nabla \boldsymbol{v}+\boldsymbol{v} \nabla)$

In order to deduce $\rho \mathrm{d} q / \mathrm{d} t$, we repeat the steps between Eq. (A2) and (A6), (with the addition of production of heat $\left.\sigma_{q}\right)$, then find that

$\rho \frac{\mathrm{d} q}{\mathrm{~d} t}=-\nabla \cdot \boldsymbol{J}_{q}^{\text {diff }}+\sigma_{q}$

where $\boldsymbol{J}_{q}^{\text {diff }}$ is the diffusion of heat, i.e., heat conduction. So,

$\rho \frac{\mathrm{d} u}{\mathrm{~d} t}=-\nabla \cdot \boldsymbol{J}_{q}^{\text {diff }}-\sum_{i} \mu_{i} \nabla \cdot \boldsymbol{J}_{n i}^{\text {diff }}-\nabla \varphi^{e} \cdot \boldsymbol{J}_{P}-\nabla \varphi^{m}$.

$\boldsymbol{J}_{M}-\nabla \varphi^{e} \cdot \boldsymbol{J}+\frac{1}{2} \boldsymbol{\sigma}:(\nabla \boldsymbol{v}+\boldsymbol{v} \nabla)+\sigma_{q}=-\nabla \cdot \boldsymbol{J}_{q}^{\text {diff }}-$

$\sum_{i} \nabla \cdot\left(\mu_{i} \boldsymbol{J}_{n i}^{\text {diff }}\right)+\sum_{i} \nabla \mu_{i} \cdot \boldsymbol{J}_{n i}^{\text {diff }}-\nabla \cdot\left(\varphi^{e} \boldsymbol{J}_{P}\right)+\varphi^{e} \nabla \cdot \boldsymbol{J}_{P}-$

$\nabla \cdot\left(\varphi^{m} \boldsymbol{J}_{M}\right)+\varphi^{m} \nabla \cdot \boldsymbol{J}_{M}-\nabla \cdot\left(\varphi^{e} \boldsymbol{J}\right)+\varphi^{e} \nabla \cdot \boldsymbol{J}+$

$\frac{1}{2}[\nabla \cdot(\boldsymbol{\sigma} \cdot \boldsymbol{v})-(\nabla \cdot \boldsymbol{\sigma}) \cdot \boldsymbol{v}+(\boldsymbol{\sigma} \cdot \boldsymbol{v}) \cdot \nabla-(\boldsymbol{\sigma} \cdot \nabla) \cdot \boldsymbol{v}]+$

$\sigma_{q}=-\nabla \cdot\left(\boldsymbol{J}_{q}^{\text {diff }}+\sum_{i} \mu_{i} \boldsymbol{J}_{n i}^{\text {diff }}+\varphi^{e} \boldsymbol{J}_{P}+\varphi^{m} \boldsymbol{J}_{M}+\varphi^{e} \boldsymbol{J}-\boldsymbol{\sigma}\right.$.

$\boldsymbol{v})+\sum_{i} \nabla \mu_{i} \cdot \boldsymbol{J}_{n i}^{\text {diff }}+\varphi^{e} \nabla \cdot \boldsymbol{J}_{P}+\varphi^{m} \nabla \cdot \boldsymbol{J}_{M}+\varphi^{e} \nabla \cdot \boldsymbol{J}-$

$(\nabla \cdot \boldsymbol{\sigma}) \cdot \boldsymbol{v}+\sigma_{q}$

Cast Eq. (A11) into the following form

$\rho \frac{\mathrm{d} u}{\mathrm{~d} t}=-\nabla \cdot \boldsymbol{J}_{u}^{\text {diff }}+\sigma_{u}$

where the internal energy flux (the diffusion part)
$\boldsymbol{J}_{u}^{\text {diff }}=\boldsymbol{J}_{q}^{\text {diff }}+\sum_{i} \mu_{i} \boldsymbol{J}_{n i}^{\text {diff }}+\varphi^{e} \boldsymbol{J}_{P}+\varphi^{m} \boldsymbol{J}_{M}$

$+\varphi^{e} \boldsymbol{J}-\boldsymbol{\sigma} \cdot \boldsymbol{v}$

and the production of internal energy

$\sigma_{u}=\sum_{i} \nabla \mu_{i} \cdot \boldsymbol{J}_{n i}^{\text {diff }}+\varphi^{e} \nabla \cdot \boldsymbol{J}_{P}+\varphi^{m} \nabla \cdot \boldsymbol{J}_{M}+\varphi^{e} \nabla \cdot \boldsymbol{J}$

$-(\nabla \cdot \boldsymbol{\sigma}) \cdot \boldsymbol{v}+\sigma_{q}$

According to Eqs. (A1), (A6), (A7) and (A13), we deduce the following

$\rho \frac{\mathrm{d} s}{\mathrm{~d} t}=-\frac{1}{T}\left[\nabla \cdot \boldsymbol{J}_{q}^{\text {diff }}+\sum_{i} \nabla \cdot\left(\mu_{i} \boldsymbol{J}_{n i}^{\text {diff }}\right)+\nabla \cdot\left(\varphi^{e} \boldsymbol{J}_{P}\right)+\nabla \cdot\right.$

$\left.\left(\varphi^{m} \boldsymbol{J}_{M}\right)+\nabla \cdot\left(\varphi^{e} \boldsymbol{J}\right)-2 \nabla \cdot(\boldsymbol{v} \cdot \boldsymbol{\sigma})\right]+\sum_{i} \frac{\mu_{i}}{T} \nabla \cdot \boldsymbol{J}_{n i}^{\text {diff }}+\frac{\nabla \varphi^{e}}{T}$.

$\boldsymbol{J}_{P}+\frac{\nabla \varphi^{m}}{T} \cdot \boldsymbol{J}_{M}+\frac{\nabla \varphi^{e}}{T} \cdot \boldsymbol{J}-\frac{\nabla \cdot(\boldsymbol{v} \cdot \boldsymbol{\sigma})}{T}-\rho \boldsymbol{F} \cdot \frac{\boldsymbol{v}}{T}=-\nabla$

$\left(\frac{\boldsymbol{J}_{q}^{\text {diff }}+\varphi^{e} \boldsymbol{J}_{P}+\varphi^{m} \boldsymbol{J}_{M}+\varphi^{e} \boldsymbol{J}-\boldsymbol{v} \cdot \boldsymbol{\sigma}}{T}\right)+\boldsymbol{J}_{q}^{\text {diff }} \cdot \nabla\left(\frac{1}{T}\right)-\sum_{i} \boldsymbol{J}_{n i}^{\text {diff }}$.

$\frac{\nabla \mu_{i}}{T}+\boldsymbol{J}_{P} \cdot \nabla\left(\frac{\varphi^{e}}{T}\right)+\boldsymbol{J}_{M} \cdot \nabla\left(\frac{\varphi^{m}}{T}\right)+\boldsymbol{J} \cdot \nabla\left(\frac{\varphi^{e}}{T}\right)-$

$\boldsymbol{\sigma}:\left[\boldsymbol{v} \nabla\left(\frac{1}{T}\right)\right]-\rho \boldsymbol{F} \cdot \frac{\boldsymbol{v}}{T}$

then we obtain the local entropy balance equation in Lagrangian form

$\rho \frac{\mathrm{d} s}{\mathrm{~d} t}=-\nabla \cdot \boldsymbol{J}_{s}+\sigma_{s}$

where the entropy flux

$\boldsymbol{J}_{S}=\frac{\boldsymbol{J}_{q}^{\text {diff }}+\varphi^{e} \boldsymbol{J}_{P}+\varphi^{m} \boldsymbol{J}_{M}+\varphi^{e} \boldsymbol{J}-\boldsymbol{v} \cdot \boldsymbol{\sigma}}{T}$

and the local entropy production

$$
\begin{gathered}
\sigma_{s}=\boldsymbol{J}_{q}^{\text {diff }} \cdot \nabla\left(\frac{1}{T}\right)-\sum_{i} \boldsymbol{J}_{n i}^{\text {diff }} \cdot \frac{\nabla \mu_{i}}{T}+\boldsymbol{J}_{P} \cdot \nabla\left(\frac{\varphi^{e}}{T}\right) \\
+\boldsymbol{J}_{M} \cdot \nabla\left(\frac{\varphi^{m}}{T}\right)+\boldsymbol{J} \cdot \nabla\left(\frac{\varphi^{e}}{T}\right)-\boldsymbol{\sigma}:\left[\boldsymbol{v} \nabla\left(\frac{1}{T}\right)\right]-\rho \boldsymbol{F} \cdot \frac{\boldsymbol{v}}{T}
\end{gathered}
$$

The two equations above are Eqs. (13) and (14).

\section{References}

[1] Y. A. Izyumov, V. N. Syromyatnikov, Phase Transitions and Crystal Symmetry. Amsterdam: Kluwer Academic Publisher, 1990.

[2] R. M. White, T. H. Geballe, Long Range Order in Solids. New York: Academic Press, 1979.

[3] A. Onuki, Phase Transition Dynamics. Cambridge: Cambridge University Press, 2002.

[4] J. I. Linares, B. Y. Moratilla, F. Ramirez, An equivalent mechanical model for representing the entropy generation in heat exchanger-application to power cycles, Int. J. Thermo., 14, 147-151, 2011.

[5] A. Sciacovelli, V. Verda, Entropy generation minimization for the optimal design of the fluid distribution system in a circular MCFC, Int. J. Thermo., 14, 167-177, 2011. 
[6] M. E. Lines, A. M. Glass, Principles and Applications of Ferroelectrics and Related Materials. New York: Oxford University Press, 1977.

[7] B. Cowan, Topics in Statistical Mechanics. London: Imperical College Press, 2005.

[8] S. T. Ai, C. T. Xu, Y. L. Wang, S. Y. Zhang, X. F. Ning, E. Noll, Comparison of and comments on two thermodynamic approaches (reversible and irreversible) to ferroelectric phase transitions, Phase Transitions, doi: 10.1080/01411590701877913.

[9] D. C. Mattis, The Theory of Magnetism Made Simple: An Introduction to Physical Concepts and to Some Useful Mathematical Methods. Singapore: World Scientific, 2006.

[10] J. R. Waldram, The Theory of Thermodynamics. Cambridge: Cambridge University Press, 1985.

[11] U. Lucia, Stationary open systems: a brief review on contemporary theories on irreversibility, Physica A, doi: 10.1016/j.physa.2012.11.027.
[12] U. Lucia, Physical model for the engineering analysis of the thermoelasticity of solid bodies, Chinese Journal of Mechanical Engineering, 13, 165-170+177, 2000.

[13] U. Lucia, G. Gervino, Hydrodynamic cavitation: from theory towards a new experimental approach, Central European Journal of Physics, doi: 10.2478/s11534-0090092-y.

[14] A. R. Allnatt, A. B. Lidiard, Atomic Transport in Solids. Cambridge: Cambridge University Press, 1993.

[15] A. Gordon, Propagation of solitary stress waves at first-order ferroelectric phase transitions, Physics Letters A, 154, 79-80, 1991.

[16] G. A. Maugin, W. Muschik, Thermodynamics with internal variables: part I. general concepts; part II. applications, Journal of Non-equilibrium Thermodynamics, 19, 217-289, 1994.

[17] D. Jou, J. Casas-Vazquez, G. Lebon, Extended Irreversible Thermodynamics, $3^{r d}$ Ed. Berlin: Springer, 2001. 